\title{
Facile fabrication of calcium-doped carbon for efficient phosphorus adsorption
}

Jishi Zhang, ${ }^{*}$ Yashan Zhang, Wenqian Zhao, Zhenmin Li, and Lihua Zang College of Environmental Science and Engineering, Qilu University of Technology (Shandong Academy of Science), Jinan 250353, China

*Email: lyzhangjishi@163.com

\section{Table of Content}

Figure S1. TG and DSC curves of CL....

Figure S2. CLDC morphologies and chemical components: CLDC400 (a), CLDC800 (b) .2

Figure S3. The influences of adsorbent concentration (a), contact time (b), initial P concentration and sorption temperature (c) on P removals and adsorption capacities (Test conditions: $5.1 \mathrm{mg} / \mathrm{L}$ of initial P, $1.2 \mathrm{~g} / \mathrm{L}$ of CLDC400 or $3.0 \mathrm{~g} / \mathrm{L}$ of CLDC 800 , sorption temperature $30^{\circ} \mathrm{C}$, contact time $5 \mathrm{~h}$ ). 3

Figure S4. Langmuir and Freundlich isotherms: (a) $30^{\circ} \mathrm{C} \mathrm{CLDC400,} \mathrm{(b)} 50^{\circ} \mathrm{C} \mathrm{CLDC400,} \mathrm{(c)} 30^{\circ} \mathrm{C}$ CLDC800, (d) $50^{\circ} \mathrm{C}$ CLDC800. . .4

Figure S5. Evaluation of adsorption kinetics: (a) pesudo-first-order kinetic model, (b) pesudo-secondorder kinetic model, (c) intraparticle diffusion model. .5 


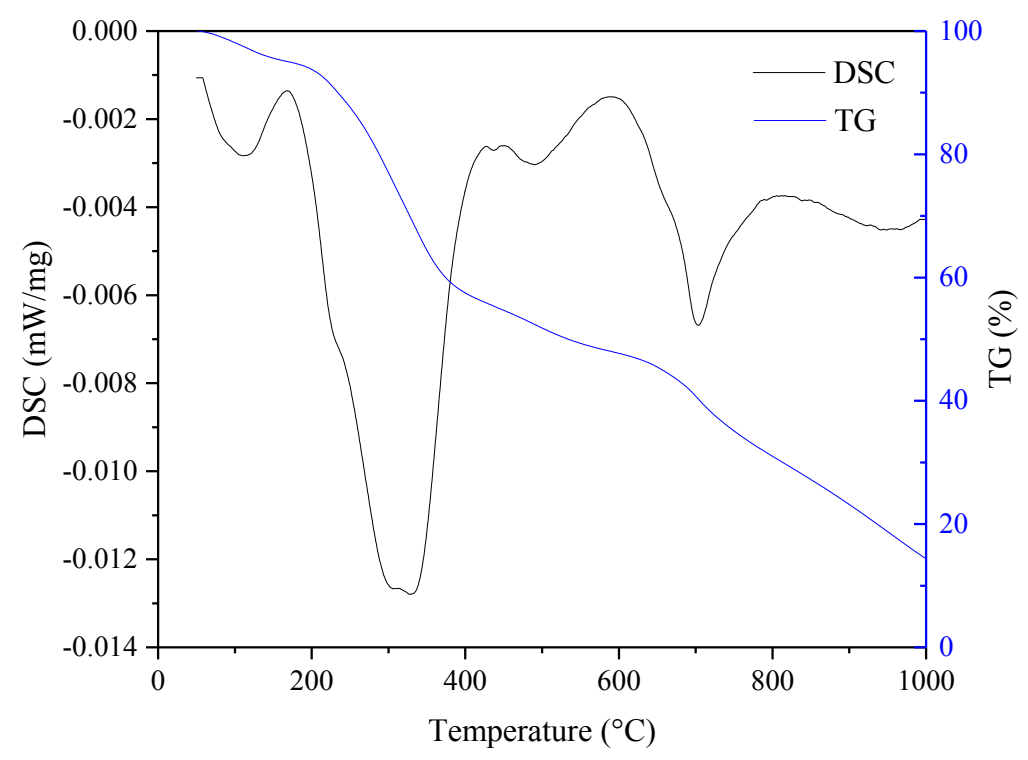

Figure S1. TG and DSC curves of CL.
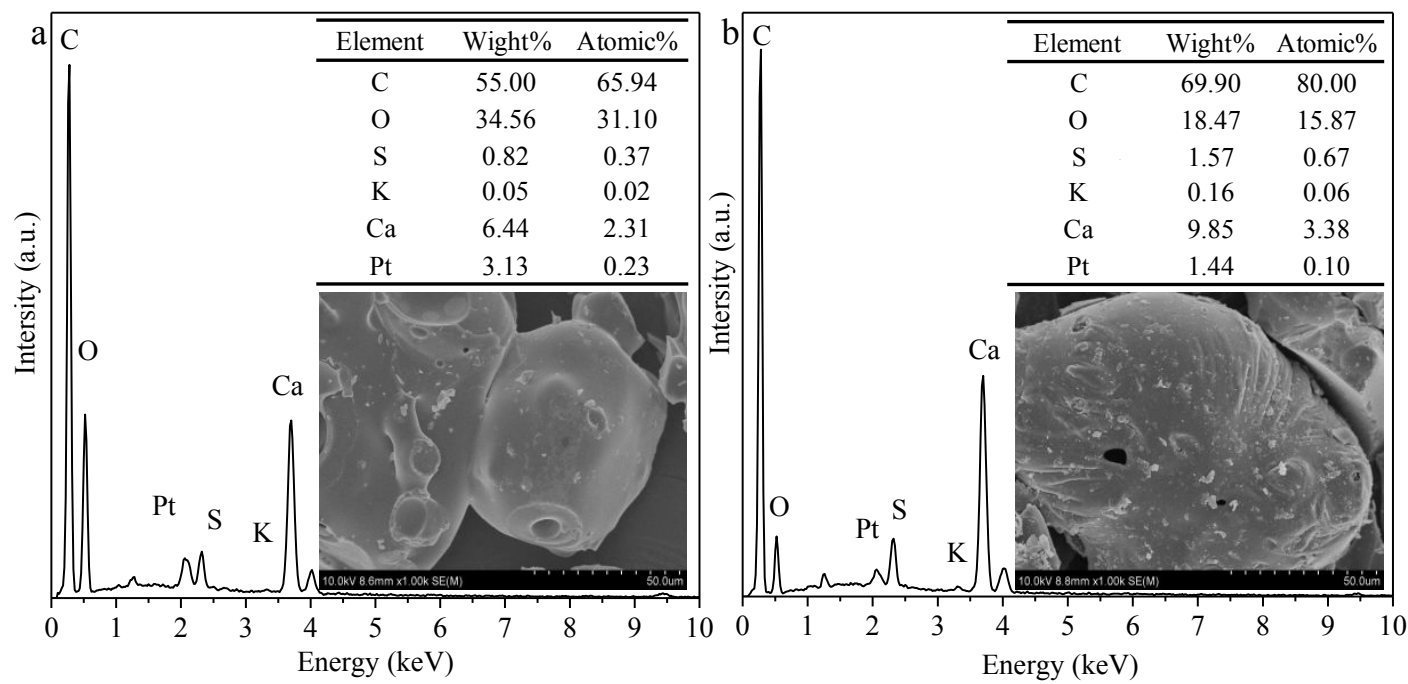

Figure S2. CLDC morphologies and chemical components: CLDC400 (a), CLDC800 (b). 

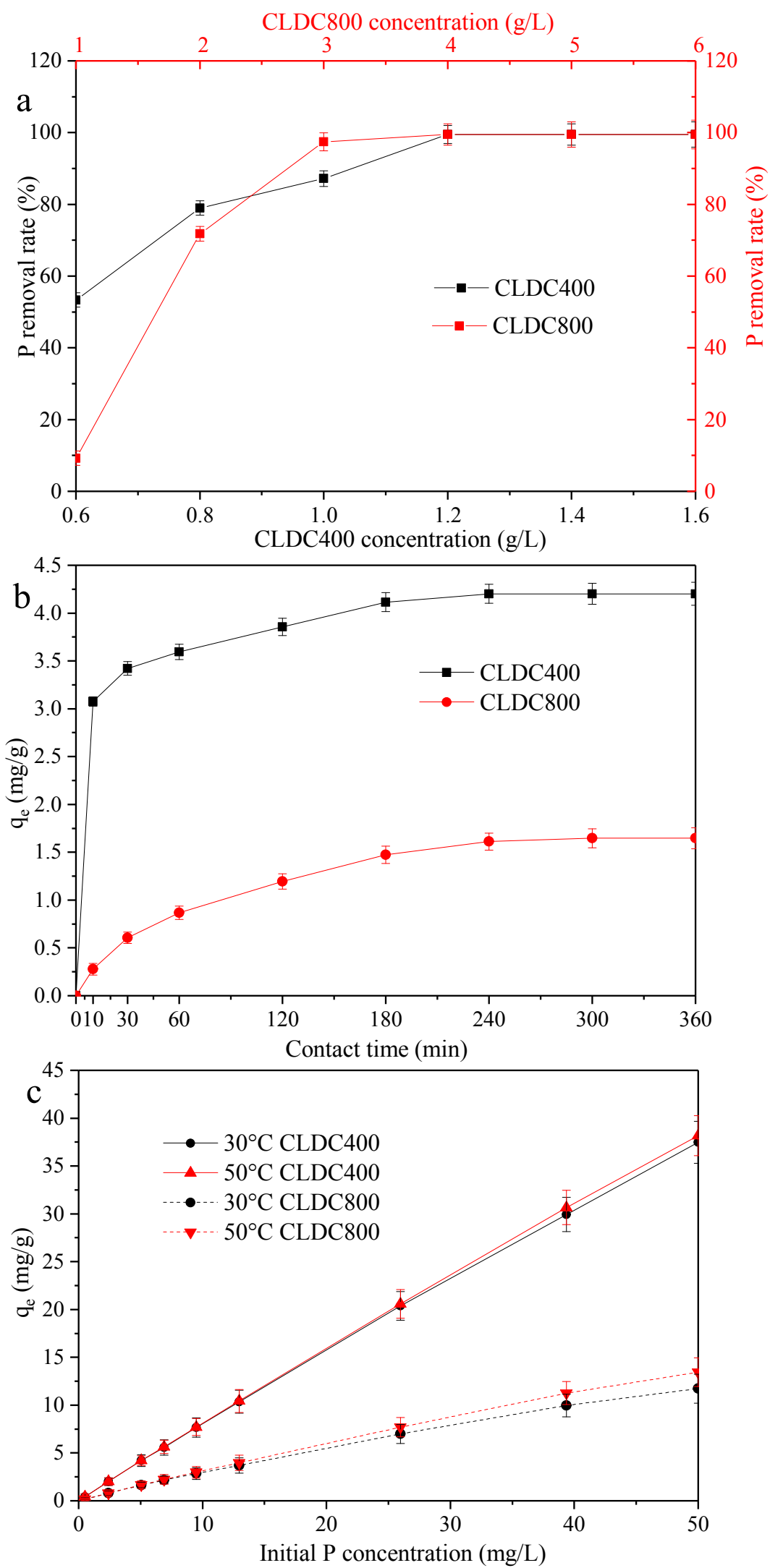

Figure S3.The influences of adsorbent concentration (a), contact time (b), initial P concentration and sorption temperature (c) on P removals and adsorption capacities (Test conditions: $5.1 \mathrm{mg} / \mathrm{L}$ of initial P, $1.2 \mathrm{~g} / \mathrm{L}$ of CLDC400 or $3.0 \mathrm{~g} / \mathrm{L}$ of CLDC800, sorption temperature $30^{\circ} \mathrm{C}$, contact time $5 \mathrm{~h}$ ). 

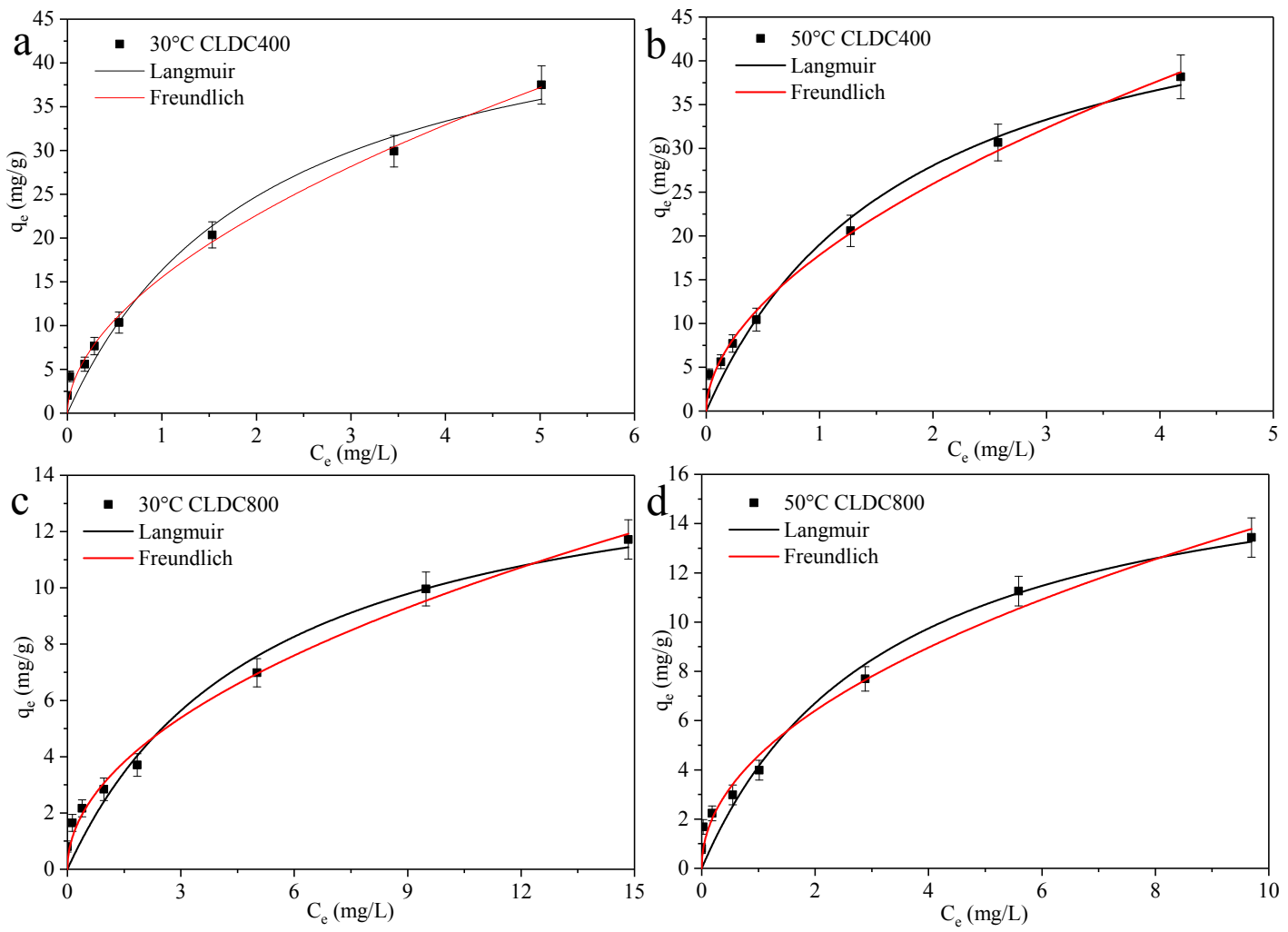

Figure S4. Langmuir and Freundlich isotherms: (a) $30^{\circ} \mathrm{C} \mathrm{CLDC400,} \mathrm{(b)} 50^{\circ} \mathrm{C} \mathrm{CLDC400,} \mathrm{(c)} 30^{\circ} \mathrm{C}$

CLDC800, (d) $50^{\circ} \mathrm{C}$ CLDC800. 

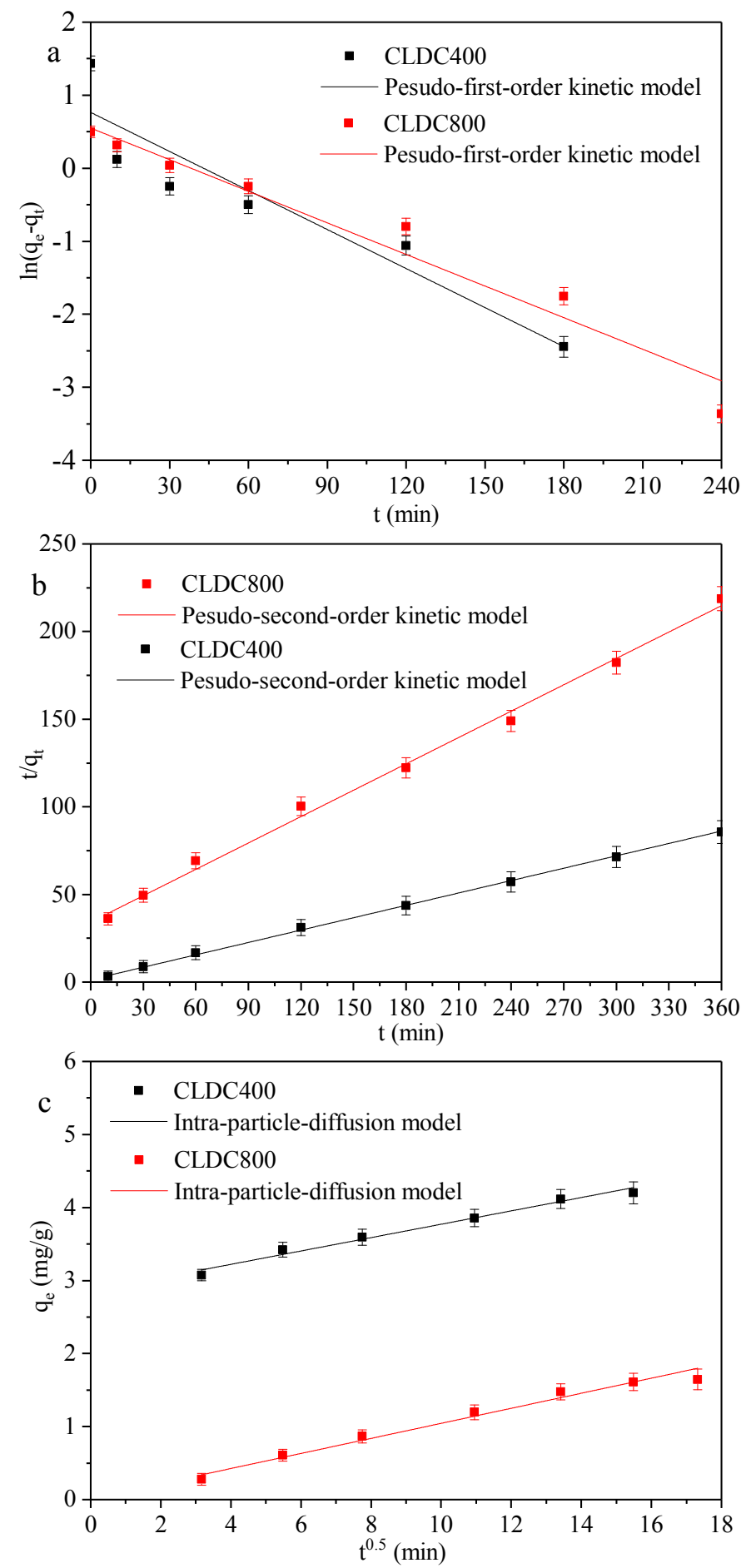

Figure S5. Evaluation of adsorption kinetics: (a) pesudo-first-order kinetic model, (b) pesudo-secondorder kinetic model, (c) intraparticle diffusion model. 